\title{
New material based on polyurethane doped with azobenzene dyes in recording media for dynamic polarization holography
}

\author{
N.A. Davidenko ${ }^{1}$, I.I. Davidenko ${ }^{1 *}$, M.Yu. Sokolov ${ }^{2}$, A.N. Gonchar ${ }^{2}$, E.V. Mokrinskaya ${ }^{1}$, S.L. Studzinsky ${ }^{1}$, \\ V.A. Pavlov ${ }^{1}$,V.V. Tarasenko ${ }^{1}$, L.S. Tonkopieva ${ }^{1}$, N.G. Chuprina ${ }^{1}$ \\ ${ }^{1}$ Taras Shevchenko Kyiv National University, \\ 64/13, Volodymyrs'ka str., 01601 Kyiv, Ukraine \\ ${ }^{2}$ Institute of Macromolecular Chemistry, NAS of Ukraine, \\ 48, Kharkivs'ke shose, 02160 Kyiv, Ukraine \\ *Corresponding author e-mail: IrynaDavydenko@gmail.com
}

\begin{abstract}
On the base of a new polyurethane with azobenzene dopants, recording media for polarization holography have been created, and their information properties have been studied when recording holograms of a plane wavefront. It has been found that the recording and relaxation times of holograms are short, and it is defined by the processes of trans-cis-isomerization of azobenzene groups without formation of the surface relief in the polymer film. The obtained results are of practical interest in the choice of photosensitive materials for holographic recording media for dynamic holography.
\end{abstract}

Keywords: azobenzene, holography, recording medium, polymeric composites.

https://doi.org/10.15407/spqeo23.01.81

PACS 42.40.Ht, 42.40.-i, 42.70.Ln

Manuscript received 16.09.19; revised version received 11.12.19; accepted for publication 18.03.19; published online 23.03.19.

\section{Introduction}

To create recording media $(\mathrm{RM})$ for polarization holography, films of polymer composites (FPCs) are used where azobenzene derivatives or related compounds are used as chromophores. The principle of holographic recording in these media with azobenzene chromophores is based on the fact that photoinduced optical anisotropy (PIA) appears upon illumination with linearly polarized light due to trans-cis-isomerization of azobenzene groups. Photoisomerization of azobenzene chromophores can cause conformational changes at the molecular level and can form stable surface reliefs in amorphous films [1-13]. These RM allow obtaining high values of the diffraction efficiency of holograms for practical application, for example, in holographic interferometry [14-16]. In this case, the recorded holograms should be stable for a long time after their registration. Another application of polarization holography is dynamic polarization holography $[17,18]$ in which RM should respond on the recorded rays only during the exposure and "forget" about the previous recording cycle. In this case, the recording and relaxation times of the hologram should be as short as possible. In these RM, polyurethanes with azobenzene fragments in the side chain can be used, as it was shown in [19] for example.
However, the literature does not sufficiently reflect the question of optimizing the choice of polyurethane and dopants from azobenzene dyes for creation of RM. This work is aimed at a comparative study of the kinetics of recording and relaxation of holograms of a plane wavefront in RM with FPC based on a new polyurethane and known polymer doped with azobenzene dyes as chromophores.

\section{Experimental}

Polyurethane (PU) based on 1,4-diphenylmethane diisocyanate, polyoxytetramethylene glycol-1000 and 1,4-butanediol, obtained by polycondensation of 1,4diphenylmethane diisocyanate with polyoxytetramethylene glycol-1000 and subsequent elongation of the resulting prepolymer chain, were synthesized. Polymethyl methacrylate (PMMA) obtained using the method of radical polymerization was used for comparison. Azobenzene dyes 4-methyl-4'-hydroxyazobenzene (Azo1) and 2-bromo-4-nitro-4'-hydroxyazobenzene (Azo2) with various donor-acceptor substitutes were used as azobenzene chromophores. A series of azobenzene dyes is chosen being based on the fact that the transition from Azo1 to Azo2 increases the dipole moment of the azobenzene chromophore, which can affect 

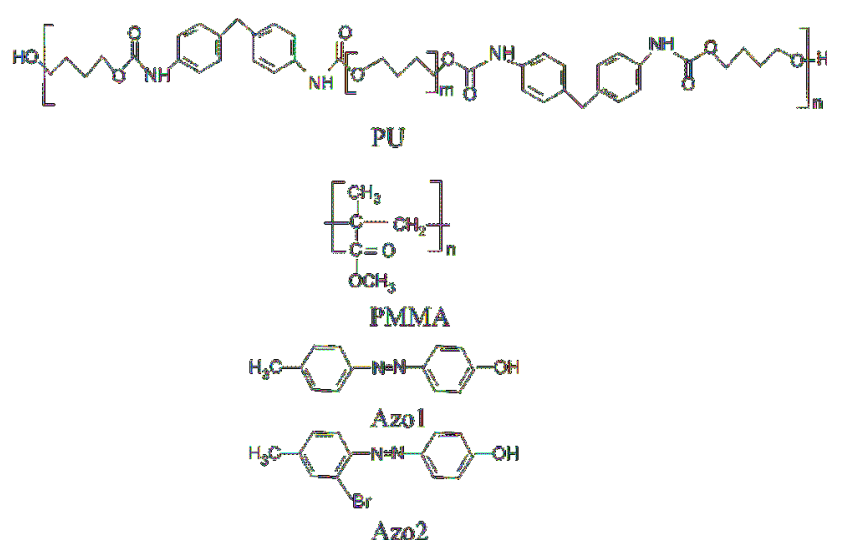

Fig. 1.

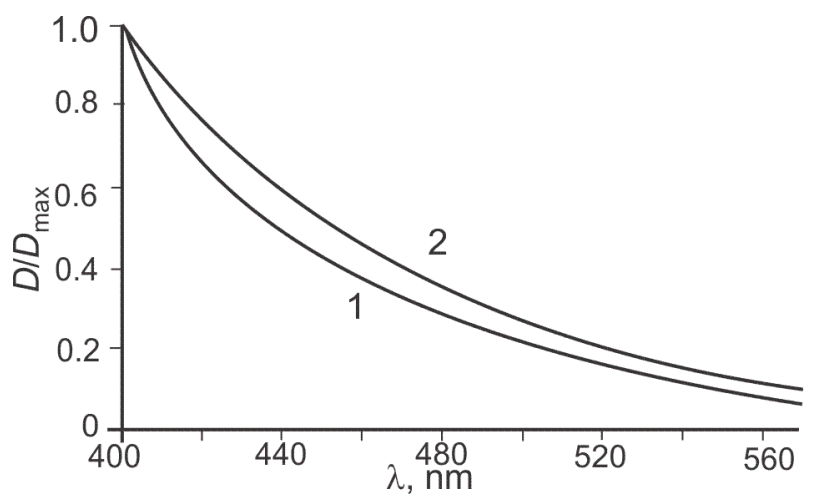

Fig. 2.

the information properties of RM. The structural formulas of the used compounds are as follows (Fig. 1).

The softening temperature of FPC based on PU and PMMA was measured by the well-known method [20], and it higher than $110{ }^{\circ} \mathrm{C}$. Therefore, it could be expected that the rheological properties of the studied FPC in RM are close between themselves.

RM samples with a free surface (glass substrate FPC) and samples of the sandwich structure (glass substrate - ITO - FPC - Ag) where ITO is the electrically conductive layer $\mathrm{SnO}_{2}: \mathrm{In}_{2} \mathrm{O}_{3}$ were prepared for research. FPC had the following composition: PU + 10 mas.\% Azo1, PU + 10 mas. $\%$ Azo2, PMMA + 10 mas. $\%$ Azo1, PMMA + 10 mas. $\%$ Azo2. FPC was prepared by watering solutions of the initial components in DMFA (for samples based on PU) and 1,2-dichloroethane (for samples based on PMMA) on glass substrates, drying for 24 hours in a heating chamber at $8{ }^{\circ} \mathrm{C}$. The thickness of FPC was $2-3 \mu \mathrm{m}$, and it was measured using an interference microscope. Samples of RM with a free surface of FPC were used to measure optical density spectra $(D)$ and to record holograms of a plane wavefront. For holographic recording, a solid-state laser with diode pumping and frequency doubling with $\lambda=532 \mathrm{~nm}$ was used with a ratio of the light intensity in the object $\left(I_{1}\right)$ and reference $\left(I_{2}\right)$ beams $1: 1$, the spatial frequency was $300 \mathrm{~mm}^{-1}$. The light intensity $I_{1}+I_{2}$ before RM was $5 \cdot 10^{3} \mathrm{~W} / \mathrm{m}^{2}$. Holograms were recorded for parallel $\left(\mathbf{e}_{1} \| \mathbf{e}_{2}\right)$ and perpendicular $\left(\mathbf{e}_{1} \perp \mathbf{e}_{2}\right)$ orientation of the electric vectors of the incident object $\left(\mathbf{e}_{1}\right)$ and reference $\left(\mathbf{e}_{2}\right)$ light waves. The diffraction efficiency $(\eta)$ of the hologram was determined according to the standard technique [21] as the ratio of the light intensity in the -1 st diffraction order to the intensity of the reference beam $I_{2}$ (also the restoring beam in the reading process) for parallel $\left(\mathbf{e}_{1} \| \mathbf{e}_{2}\right)$ and perpendicular $\left(\mathbf{e}_{1} \perp \mathbf{e}_{2}\right)$ orientation of the electric vectors of the recording light waves (respectively, as $\eta_{\|}$and $\eta_{\perp}$ ). The dependences $\eta_{\|}(t)$ and $\eta_{\perp}(t)$ on time $(t)$ were measured after the start and end of the hologram exposure. The maximal diffraction efficiency $\eta_{\| \max }$ and $\eta_{\perp \max }$ as well as the recording $\left(t_{1}\right)$ and relaxation $\left(t_{2}\right)$ times of holograms were measured. The values of $t_{1}$ and $t_{2}$ were estimated, respectively, by using the value of $\eta_{\| \max } / 2$ after the start of hologram registration and $\eta_{\perp \max } / 2$ after the end of it. Each new measurement was carried out on a new area of RM to eliminate the influence of the previous experiment (memory of the hologram recording). In the samples of sandwich structure, the dielectric characteristics of FPC were measured: the values of the tangent of dielectric loss angle $(\operatorname{tg} \delta)$ and electric capacitance $(C)$ depending on the frequency $(f)$ of a sinusoidal alternating electric voltage with the amplitude $5 \mathrm{~V}$. The measurement results were averaged over 3 samples of identical RM. All measurements were carried out at room temperature close to $20^{\circ} \mathrm{C}$.

\section{Results and discussion}

Fig. 2 shows the normalized optical density spectra of FPCs with Azo1 and Azo2. Within the visible spectral range, the absorption of these FPCs is determined by the longwave absorption edge of the azobenzene chromophores, and, therefore, the FPC spectra based on PU and PMMA almost coincide. The bathochromic shift in FPC with Azo2, as compared to Azo1, is caused by the presence of electron-acceptor substitutes in Azo2 increasing the dipole moment of the Azo2 azobenzene dye molecule as compared to Azo1.

In RM with FPC, holograms of a plane wavefront are recorded for both orientations of electric vectors $\mathbf{e}_{1} \| \mathbf{e}_{2}$ and $\mathbf{e}_{1} \perp \mathbf{e}_{2}$. The main results of measuring $\eta_{\| \max }$ and $\eta_{\perp \text { max }}$ as well as the kinetics of recording and relaxation of holograms are presented in Table and Fig. 2.

From these results, it is clear that in RM with FPC based on PMMA, the relation $\eta_{\perp \max }>\eta_{\| \max }$ found before for RM based on other azobenzene-containing polymers $[3,5,12,13]$ is fulfilled. A peculiarity of the dependences $\eta_{\|}(t)$ and $\eta_{\perp}(t)$ in the studied RM is the presence of at least two kinetic sections: fast and slow. After the start of the hologram exposure, $\eta_{\|}(t)$ and $\eta_{\perp}(t)$ rapidly increase, and then this growth slows down. The same regularity is observed after turning off the hologram recording. However, in FPC based on the new PU $\eta_{\perp \max } \leq \eta_{\| \max }$ and all the recording and relaxation 
Table. Measurement results for $\eta_{\| \max }$ and $\eta_{\perp \max }$ in RM with FPC immediately after their exposure for $2 \mathrm{~min}$.

\begin{tabular}{|c|c|c|}
\hline FPC & $\begin{array}{c}\eta_{\| \max } \\
t_{1}, \mathrm{~s} \\
t_{2}, \mathrm{~s} \\
\end{array}$ & $\begin{array}{c}\eta_{\perp \max } \\
t_{1}, \mathrm{~s} \\
t_{2}, \mathrm{~s} \\
\end{array}$ \\
\hline PU + 10 mas. $\%$ Azo1 & $\begin{array}{c}3 \cdot 10^{-4} \\
2 \\
1 \\
\end{array}$ & $\begin{array}{l}5 \cdot 10^{-5} \\
<0.1 \\
<0.1\end{array}$ \\
\hline PU + 10 mas. $\%$ Azo 2 & $\begin{array}{l}7 \cdot 10^{-5} \\
<0.1 \\
<0.1\end{array}$ & $\begin{array}{l}6 \cdot 10^{-5} \\
<0.1 \\
<0.1 \\
\end{array}$ \\
\hline $\begin{array}{c}\text { PMMA + } 10 \text { mas. } \% \\
\text { Azo1 }\end{array}$ & $\begin{array}{c}2 \cdot 10^{-4} \\
10 \\
15 \\
\end{array}$ & $\begin{array}{c}2.5 \cdot 10^{-4} \\
10 \\
15 \\
\end{array}$ \\
\hline $\begin{array}{c}\text { PMMA + } 10 \text { mas. } \% \\
\text { Azo } 2\end{array}$ & $\begin{array}{c}3.5 \cdot 10^{-5} \\
15 \\
20\end{array}$ & $\begin{array}{c}7.8 \cdot 10^{-5} \\
15 \\
20\end{array}$ \\
\hline
\end{tabular}

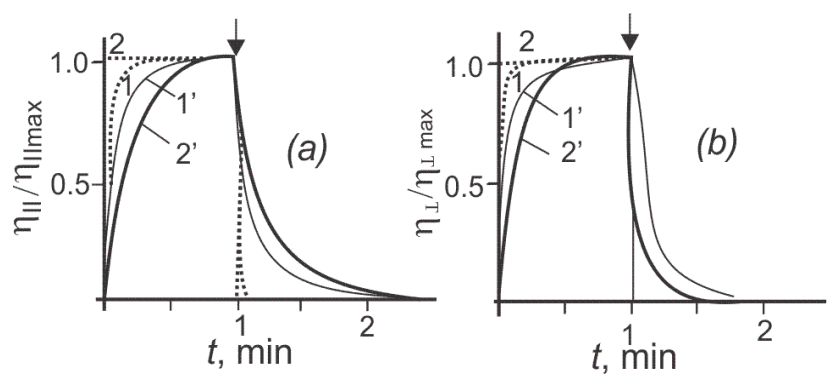

Fig. 3.

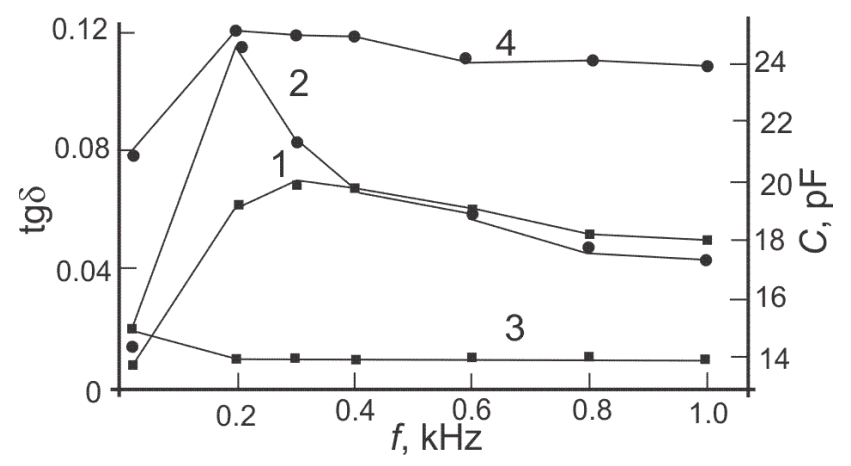

Fig. 4.

processes of holograms occur much faster as compared to FPC based on PMMA (Table, Fig. 3), and these two sections in $\eta_{\|}(t)$ and $\eta_{\perp}(t)$ dependences are not observed. In this case, the fastest change of the diffraction efficiency of holograms is observed in RM with FPC and Azo2. Thus, an increase of the dipole moment of azobenzene chromophore molecules in both FPCs based on PMMA and on PU contributes to an increase of diffraction efficiency. In RM based on PU, an increase of the dipole moment of azobenzene chromophore molecules is also accompanied by a decrease of $t_{1}$ and $t_{2}$. The latter means that formation and relaxation of FIA in FPC based on PU occurs more quickly than in FPC based on PMMA. To clarify the possible reasons for this difference in the properties of FPCs based on PU and PMMA, we carried out additional investigations of the dielectric characteristics inherent to these FPCs. Fig. 4 presents the main results of these studies - the plots of the dependences of $\operatorname{tg} \delta$ and $C$ on $f$. From these experiments, the values of dielectric function $\varepsilon$ of FPC were calculated, which for PMMA and PU are equal to 3.3 and 6.7 , respectively.

It is known [4-11] that the main mechanism for recording polarization holograms with a long relaxation time (storage) is trans-cis-isomerization of azobenzene fragments under action of linearly polarized light from the absorption range of isomers of the azobenzene chromophore. At the same time, rearrangement of a polymeric matrix can occur. This rearrangement of the polymeric matrix is responsible for the long-term storage of holographic recording. When $\mathbf{e}_{1} \| \mathbf{e}_{2}$ and $\mathbf{e}_{1} \perp \mathbf{e}_{2}$, the azobenzene groups involved in photoisomerization are oriented in the respective directions and additional deforming forces appear acting on the main polymer chain and resulting in formation of a geometric relief of the FPC surface in RM. Since liquidation of the surface relief of a film occurs more slowly than relaxation of a latent holographic image in polarization-sensitive RM, the presence of such a relief generally increases the relaxation time of $\eta$. Similar structures on the surface of FPC are formed during recording the relief amplitude holograms by photothermoplastic method on photoconductive polymer films [22]. Homogeneity of these structures provides high informational characteristics of RM and long-term storage of holographic recording. However, after holographic recording in the investigated samples of RM with FPC based on PU and PMMA, we were not able to register a change in the topography of the FPC surface. We tried to do this using a previously developed technique [12] employed an interference microscope. Therefore, we can assume that when recording holograms in the studied RM, a stable surface relief of FPC is not formed.

\section{Conclusions}

The differences in the recording and relaxation velocity of polarization holograms in FPC based on new PU as compared to the known polymer are not related to the rheological properties of these polymers, since the geometrical relief of the surface of these FPCs is not conserved after recording the holograms. As the dielectric function $\varepsilon$ of PU is more than 2 times higher than $\varepsilon$ of PMMA (Fig. 4) the differences in information properties of RM based on these polymers are obviously related to their dielectric properties. To clarify the effect 
of dielectric properties of the polymer matrix on the information properties of RM, additional studies are needed, which will be the aim of our next works. The results of experimental investigations with a new polyurethane as the basis of FPC for RM allow us to conclude that this PU is of practical interest in the development and creation of new recording media for dynamic polarization holography.

\section{References}

1. Bian S., Williams J.M., Kim D.Yu., Li L., Balasubramanian S., Kumar J., Tripathy S. Photoinduced surface deformations on azobenzene polymer films. J. Appl. Phys. 1999. 86. P. 44984502. https://doi.org/10.1063/1.371393.

2. Barachevsky V.A. Current status of develoment of light-sensitive media for holography (a review). Optics and Spectroscopy. 2018. 124. P. 373-407. https://doi.org/10.1134/S0030400X18030062.

3. Davidenko N.A., Davidenko I.I., Kravchenko V.V., Marinin A.I., Mokrinskaya E.V., Pavlov V.A., Tarasenko V.V., Chuprina N.G. Recording polarization holograms in films of 4-((2-bromo-4nitrophenyl)diazenyl)phenyl methacrylate copolymers. Optics and Spectroscopy. 2019. 126. P. 135139. https://doi.org/10.1134/S0030400X19020103.

4. Priimagi A., Shevchenko A.J. Azopolymer-based micro- and nanopatterning for photonic applications. Polymer Sci. Part B: Polymer Phys. 2014. 52. P. 163-182.

https://doi.org/10.1002/polb.23390.

5. Davidenko N.A., Davidenko I.I., Pavlov V.A., Chuprina N.G., Tarasenko V.V., Studzinsky S.L. Adjustment of diffraction efficiency of polarization holograms in azobenzene polymer films using electric fields. J. Appl. Phys. 2017. 122. P. 0131011-6. https://doi.org/10.1063/1.4990995.

6. Natansohn A., Rochon P. Photoinduced motion in azo-containing polymers. Chem. Rev. 2002. 102. P. 4139-4175. https://doi.org/10.1021/cr970155y.

7. Simonov A.N., Uraev D.V., Kostromin S.G., Shibaev V.P., Stakhanov A.I. Polarizationcontrolled optical recording in azocontaining amorphous polymer films. Laser Physics. 2002. 12. P. 1294-1302.

8. Emoto A., Uchida E., Fukuda T. Optical and physical applications of photocotrollable materials: azobenzene-containing and liquid crystalline polymers. Polymers. 2012. 4. P. 150-186.

https://doi.org/10.3390/polym4010150.

9. Garrot D., Lassailly Y., Lahlil K., Boilot J.P., Peretti J. Real-time near-field imaging of photoinduced material motion in thin solid films containing azobenzene derivatives. Appl. Phys. Lett. 2009. 94. P. 033303-1-3. https://doi.org/10.1063/1.3073742.

10. Zhou J., Yang J., Ke Y., Shen J., Zhang Q., Wang $\mathrm{K}$. Fabrication of polarization grating and surface relief grating in crosslinked and non-crosslinking azopolymer by polarization holography method. Optical Materials. 2008. 30. P. 1787-1895. https://doi.org/10.1016/j.optmat.2007.08.011.

11. Häckel M., Kador L., Kropp D., Schmidt H.-W. Polymer blends in azobenzene-containing block copolymers in stable rewritable holographic media. Adv. Mater. 2007. 19. P. 227-231. https://doi.org/10.1002/adma.200601458.

12. Davidenko N.A., Davidenko I.I., Pavlov V.A., Chuprina N.G., Kravchenko V.V., Tarasenko V.V., Studzinsky S.L., Mokrinskaya E.V., Tonkopieva L.S. Recording media for polarization holography with diffraction efficiency adjusted using electric field. Optik - International Journal for Light and Electron Optics. 2018. 158. P. 1308-1312. https://doi.org/10.1016/j.ijleo.2018.01.018.

13. Davidenko N.A., Davidenko I.I., Pavlov V.A., Chuprina N.G., Mokrinskaya E.V., Tarasenko V.V., Tonkopieva L.S., Kravchenko V.V. Recording medium based on the azobenzene copolymer with free surface and in sandwich-structure for polarization holography. Optical Materials. 2018. 76. P. 169-173.

https://doi.org/10.1016/j.optmat.2017.12.027.

14. Nikolova L., Ramanujam P.S. Polarization Holography. Cambridge, UK: Cambridge University Press, 2009.

15. Yoshimura T. Thin-Film Organic Photonics: Molecular Layer Deposition and Applications. Boca Raton - London - New York: CRC Press, 2011.

16. Naydenova I. (Ed.). Holograms - Recording Materials and Applications. Intech: Rijeka, Croatia, 2011.

17. Romanov O.G., Gorbach D.V., Tolstik A.L. Transformations of optical vortices by polarization dynamic holograms. Optics and Spectroscopy. 2013. 115. P. 335-339.

18. Kabanov V.V., Rubanov A.S., Tolstik A.L., Chaley A.V. Optical multistability of four-wave mixing in a resonant medium. Opt. Commun. 1989. 71. P. 219-223. https://doi.org/10.1016/0030-4018(89)90431-8.

19. Goldenberg L.M., Kulikovsky L., Kulikovska O., Stumpe J. New materials with detachable azobenzene: effective, colourless and extremely stable surface relief gratings. J. Mater. Chem. 2009. 19. P. 8068-8071. https://doi.org/10.1039/B918130J.

20. Schwetlick K. Organicum. WILEY-VCH Verlag GmbH, 2001.

21. Collier R.J., Burckhart C.B., Lin L.H. Optical Holography. NY. and London: Academic Press, 1973.

22. Davidenko N.A., Davidenko I.I., Pavlov V.A., Chuprina N.G., Kravchenko V.V., Kuranda N.N., Mokrinskaya E.V., Studzinsly S.L. Photothermoplastic recording media and its application in the holographic method of determination of refracttive index of liquid objects. Appl. Opt. 2018. 57. P. 1832-1837. https://doi.org/10.1364/AO.57.001832. 